\title{
Safety of Daily Albuterol in Infants with a History of Bronchospasm: A Multi-Center Placebo Controlled Trial ${ }^{\S}$
}

\author{
James A. Hedrick ${ }^{1}$, James W. Baker ${ }^{2}$, Arthur B. Atlas ${ }^{3}$, Aftab A. Naz ${ }^{4}$, \\ William R. Lincourt ${ }^{*}$, , Roopa Trivedi ${ }^{5}$ Anna Ellworth ${ }^{5}$ and \\ Angela M. Davis ${ }^{5}$
}

\author{
${ }^{1}$ Kentucky Pediatric/Adult Research, 201 South $5^{\text {th }}$ Street, Suite 102; Bardstown, Kentucky 40004, USA \\ ${ }^{2}$ Allergy, Asthma and Dermatology Research Center, LLC, 3975 SW Mercantile Drive, Suite 165; Lake Oswego, Oregon \\ 97035, USA \\ ${ }^{3}$ Morristown Memorial Hospital, 100 Madison Avenue, Morristown, New Jersey 07960, USA \\ ${ }^{4}$ Madera Family Medical Group, 1111 West $4^{\text {th }}$ Street, Madera, California 93637, USA \\ ${ }^{5}$ GlaxoSmithKline, 5 Moore Drive, Research Triangle Park, North Carolina 27709, USA
}

\begin{abstract}
Introduction: Inhaled short-acting bronchodilators are recommended for the quick relief of bronchospasm symptoms in children including those less than five years of age. However, limited safety data is available in this young population.
\end{abstract}

\begin{abstract}
Methods: Safety data were analyzed from a randomized, double-blind, parallel group, placebo-controlled multicenter, study evaluating albuterol HFA $90 \mu \mathrm{g}$ or $180 \mu \mathrm{g}$ versus placebo three times a day for 4 weeks using a valved holding chamber, Aerochamber Plus and facemask in children birth $\leq 24$ months old with a history of bronchospasm.
\end{abstract}

Results: The overall incidence of adverse events (AE) during treatment was: albuterol $90 \mu \mathrm{g}(59 \%)$, albuterol 180 $\mu \mathrm{g}(76 \%)$ and placebo (71\%). The most frequently reported AEs were pyrexia in $7(24 \%), 2(7 \%)$, and $3(11 \%)$ subjects in the albuterol $180 \mu \mathrm{g}$, albuterol $90 \mu \mathrm{g}$, and placebo groups, respectively. Upper respiratory tract infection (URTI) occurred in 5 $(17 \%)$ and $3(11 \%)$ subjects in the albuterol $180 \mu \mathrm{g}$ and placebo groups, respectively. Sinus tachycardia occurred in 5 $(17 \%), 2(7 \%)$ and $2(7 \%)$ subjects receiving albuterol $180 \mu \mathrm{g}$, albuterol $90 \mu \mathrm{g}$ and placebo, respectively. One subject in each of the albuterol treatment groups experienced drug related agitation and/or restlessness or mild sinus arrhythmia. No drug-related QT prolongation or abnormal serum potassium and glucose levels were reported in the albuterol treatment groups.

Conclusion: This study provides additional albuterol HFA safety information for the treatment of children aged birth $\leq 24$ months with a history of bronchospasm.

\section{INTRODUCTION}

Asthma is an increasingly important cause of chronic morbidity affecting approximately $5.5 \%$ children less than 5 years of age in the US, with $3.9 \%$ of this age group experiencing an asthma attack in the last 12 months $[1,2]$. This high morbidity has a significant impact in the health care system, with asthma in children alone accounting for almost 3 million physician visits and 200,000 hospitalizations each year [3].

*Address correspondence to this author at the GlaxoSmithKline, 5 Moore Drive, Research Triangle Park, NC 27709, USA; Tel: (919) 483-6284; Fax: (919) 483-4300; E-mail: william.r.lincourt@gsk.com

${ }^{\S}$ Originally presented in abstract form at the Annual Meeting of the American Academy of Allergy Asthma and Immunology, March 3-7, 2006, Miami Beach, FL, USA.
Albuterol, a short acting beta $2_{2}$ adrenoceptor agonist, is widely used for rapid relief of respiratory symptoms in accordance with guidelines for current asthma management which includes treatment recommendations for children less than five years of age $[4,5]$. Treatment of symptoms in this very young population can be challenging. In the past, the use of nebulized therapy was the preferred method in treating asthma symptoms in young children. However, many studies including those in children less than 5 years of age have demonstrated efficacy, cost-effectiveness and convenience of an albuterol MDI used with a spacer or valved holding chamber (VHC) [6-11].

Currently there are limited approved treatment options for reversible obstructive airway disease in children less than 4 years of age. An earlier study in children ages 24 to $\leq 48$ months with symptoms of reversible obstructive airway disease demonstrated no clinically relevant safety signals 
[12]. However, clinical data in children younger than 24 months is limited. Therefore the current study was conducted primarily to evaluate the safety of albuterol HFA $90 \mu \mathrm{g}$ or $180 \mu \mathrm{g}$ (VENTOLIN HFA is a registered trade name of GlaxoSmithKline, Research Triangle Park, NC) administered three times daily via a MDI and an Aerochamber Plus VHC (Aerochamber Plus is a registered trade name of Monaghan Medical, Plattsburgh, NY, USA) in symptomatic children between birth and 24 months of age.

\section{METHODS}

\section{Study Population}

Patients were recruited from 33 family practice, allergy, and pediatric practices in the US. The study (SBO30001) was performed in accordance with the ethical principles in the Declaration of Helsinki and was consistent with the International Conference on Harmonization/Good Clinical Practice and applicable regulatory requirements. The protocol was approved by the Coast Independent Review Board in San Clemente, CA and other center institutional review boards as appropriate and each patient's parent or legal guardian provided a signed consent before study procedures were undertaken.

All children were assessed by the investigators to ensure that they met all study eligibility criteria; birth to $<24$ months old, a history of symptoms of bronchospasm that required outpatient care, and had received regular maintenance asthma pharmacotherapy (except systemic corticosteroids) for 3 weeks prior to Visit 1 and/or a short-acting $\beta_{2}$-agonist prior to Visit 1 . In addition all patients were required to be symptomatic and to use albuterol on at least 2 of 7 consecutive days during the screening period. Subjects experiencing an exacerbation (defined as an emergency room visit, hospitalization, an unscheduled doctor visit/contact requiring additional treatment with an asthma medication or increased use of patient's maintenance asthma medication other than rescue albuterol) during screening were not eligible. Subjects were excluded if they had a history of lifethreatening asthma; other severe concurrent disease; a current upper or lower respiratory tract or middle ear infection; recent systemic corticosteroid use; premature birth (before 34 weeks of gestation); or clinically significant laboratory or ECG abnormalities. Leukotriene modifiers, cromolyn and/or nedocromil and inhaled corticosteroids were allowed if started prior to screening and remained unchanged during the study. All other asthma medications were not allowed during the study.

\section{Study Design}

This randomized, double-blind, parallel-group, placebocontrolled study began with a 7 to 28 -day screening period, followed by a 29-day treatment period. Subjects were randomized to receive albuterol inhalation aerosol $90 \mu \mathrm{g}$ or $180 \mu \mathrm{g}$ or placebo HFA three times daily for a total of 29 days via a MDI in conjunction with a valved holding chamber, the Aerochamber Plus and attached face mask (small or medium size). Each subject was dispensed two MDI inhalers both containing either albuterol HFA or placebo HFA, a holding chamber, face mask (small or medium) and administration instructions. Parents/guardians were asked to have their child inhale one puff from each of the two inhalers three times daily, approximately 4 to 6 hours apart for 29 days. Open-label albuterol HFA MDI (90 $\mu$ g/actuation) with a holding chamber other than the one used for study drug or albuterol nebule $(2.5 \mathrm{mg} / 3 \mathrm{~mL})$ formulations, were provided for rescue use as needed. Study medication and rescue albuterol were not permitted for at least 4 hours prior to the procedures on Days 1 and 29 during which ECGs were performed before and after study medication was given in the clinic. Subjects attended the clinic during treatment evaluations on Days 1, 8, 15 and 29.

The primary goal of this study was to evaluate the safety of albuterol inhalation aerosol in patients $<24$ months of age. Safety measures included the assessment of adverse events, signs and symptoms of adrenergic stimulation (assessed by certain questions on the Functional Status II (R) questionnaire (FSII(R)), [13] the daily diary, and heart rate), clinical laboratory assessments for serum potassium and glucose, ECG measurements (including QTc interval) and physical examination including vital signs. An independent electrocardiographer, blinded to treatment assignments interpreted the ECGs. The cut-off for the QTc interval was chosen as $460 \mathrm{msec}$ based on suggested QTc values $\geq 460$ msec as prolonged in children aged 1-15 years.

Blood samples were collected on day 1 and day 29 . Serum potassium and glucose were evaluated by a centralized laboratory (Quest Laboratory). Safety was assessed by the frequency of AEs, abnormal laboratory, ECGs evaluating heart rate, QT/QTc interval, blood pressure and other ECG interval measurements and any signs and symptoms of adrenergic stimulation. Each investigator was required to determine the causality assessment of the relationship of the event to the study drug and whether it constituted a serious adverse event.

Twenty-four hour daily asthma symptom scores were documented by the patient's care-giver in the diary record provided at the screening visit. Other efficacy parameters also noted in the diary record included 24-hour rescue albuterol use, nighttime airway symptom scores, percentage of symptom-free 24-hour days, daytime symptom scores, percentage of nights with no awakenings due to symptoms requiring albuterol treatment, use of rescue systemic corticosteroid, and/or inhaled corticosteroids during the study, and the number of exacerbations. Study personnel checked the diary record for completion and compliance at all clinic visits.

Study medication was supplied to the investigators as 2 inhalers (double blind canisters labeled Can A and Can B) for administration via MDI with HFA propellants, with instructions to test spray each MDI four times before use the first time and inserting into holding chamber. Rescue medication was provided separately and all study medication use was documented in a patient diary record provided at study start. Patient guardians were advised to return all clinical supplies after each visit. Treatment compliance was calculated for each subject as a proportion of the number of doses used per expected number of doses used for the double-blind treatment period.

\section{Statistical Analysis}

Approximately 80 subjects ( 25 per treatment group) were enrolled, so that at least 22 subjects per treatment group 
would complete the study. This study was conducted primarily to assess safety, thus the sample size was not expected to provide enough statistical power to detect significant differences in the efficacy parameters between any two treatment arms. Analyses of the efficacy endpoints and FSII(R) total scores were performed for descriptive purposes only. The primary efficacy measure was mean change from baseline to the endpoint (the average over the 4week treatment period) in 24-hour daily asthma symptom scores. Symptoms including cough, wheeze, and shortness of breath were rated by the parent/guardian on a scale of 0 (no symptoms) to 3 (severe symptoms that restricted normal daily activities or kept patient awake most of the night). Other measures included 24-hour rescue albuterol use, day and nighttime asthma symptom scores, percentage of symptom-free 24-hour days, nighttime awakenings due to symptoms, use of rescue systemic or inhaled corticosteroids, and the number of subjects with exacerbations.

All analyses were conducted on the intent-to-treat (ITT) population, which comprised all randomized subjects who received at least one dose of study medication. Two-sided statistical tests with a 0.05 level of significance were used and $95 \%$ confidence intervals were provided. Analyses of 24-hour asthma symptom scores, and FSII(R) total scores were performed using analysis of covariance, with baseline, age, gender, and region (grouping of investigational sites by geographic location), and concurrent medication use for asthma (subjects receiving a fixed dose of inhaled corticosteroids and/or leukotriene modifiers, or not) as the covariates, in addition to treatment effect. No inferential statistics were provided for any of the other efficacy measures. Twenty-four hour asthma symptom scores were summarized by concurrent medication use for asthma. FSII(R) total scores were summarized for those subjects for whom the same parent/guardian completed the questionnaire at all visits. No statistical testing was conducted on safety measures, which were summarized using descriptive statistics. All statistical analyses were performed using SAS (SAS Institute, Cary, NC) software version 8.

\section{RESULTS}

\section{Patient Demographics and Disposition}

A total of 118 subjects were screened for this study, of which 32 were not randomized due to failure to meet entry criteria, including not meeting rescue albuterol use requirements or symptom criteria prior to Visit 2, a current respiratory infection, lack of compliance or withdrawing consent; or an adverse event. Demographic characteristics of the 86 randomized subjects were similar across all groups (Table 1). The mean age of onset of asthma symptoms was 5.5 months, and the most commonly identified risk factors for asthma were family history of asthma (94\%), history of eczema $(24 \%)$, year round hay fever $(15 \%)$, seasonal hay fever $(15 \%)$ and history of food allergy and history of sinus infection (each at 13\%). Very few subjects $(4,1$ and 3 subjects in the placebo and albuterol HFA $90 \mu \mathrm{g}$ and $180 \mu \mathrm{g}$ groups, respectively) discontinued from the study (Table 1). Approximately half of the subjects in each treatment group used concurrent asthma medications. Budesonide and montelukast use were most frequently reported. Mean compliance with study medication was high in all groups (89.5-92\%).

\section{Safety}

A total of 59 subjects $(69 \%)$ reported at least one adverse event, [20 (71\%), $17(59 \%)$ and $22(76 \%)$ subjects in the placebo, albuterol HFA $90 \mu \mathrm{g}$ and $180 \mu \mathrm{g}$ groups, respectively]. The most frequently reported events were pyrexia or fever $(3,2$, and 7 subjects in the placebo, albuterol HFA $90 \mu \mathrm{g}$ and $180 \mu \mathrm{g}$, groups, respectively), nasopharyngitis $(3,2$ and 4 subjects in the placebo, albuterol HFA $90 \mu \mathrm{g}$ and

Table 1. Demographics and Disposition of Subjects

\begin{tabular}{|c|c|c|c|}
\hline & $\begin{array}{l}\text { Placebo } \\
(N=28)\end{array}$ & $\begin{array}{c}\text { Albuterol HFA } \\
90 \mu \mathrm{gID}(N=29)\end{array}$ & $\begin{array}{c}\text { Albuterol HFA } \\
180 \mu \mathrm{g} T I D(N=29)\end{array}$ \\
\hline \multicolumn{4}{|l|}{ Gender } \\
\hline Male, n (\%) & $19(68)$ & $19(66)$ & $21(72)$ \\
\hline Female, n (\%) & $9(32)$ & $10(34)$ & $8(28)$ \\
\hline Age, mean months (range), & $13.9(3-23)$ & $14.1(3-22)$ & $16.3(6-23)$ \\
\hline \multicolumn{4}{|l|}{ Ethnic origin, $\mathrm{n}(\%)$} \\
\hline White & $15(54)$ & $17(59)$ & $13(45)$ \\
\hline American Hispanic & $6(21)$ & $7(24)$ & $9(31)$ \\
\hline Black & $7(25)$ & $5(17)$ & $6(21)$ \\
\hline Other & 0 & 0 & $1(3)$ \\
\hline Concurrent asthma medications, $\mathrm{n}(\%)$ & $15(54)$ & $14(48)$ & $15(52)$ \\
\hline Number Discontinued from Study, n (\%) & $4(14)$ & $1(3)$ & $3(10)$ \\
\hline Due to Lack of efficacy & $4(14)$ & 0 & $2(7)$ \\
\hline Due to Consent Withdrawn & 0 & $1(3)$ & 0 \\
\hline Due to Other & 0 & 0 & $1(3)$ \\
\hline
\end{tabular}

HFA, hydrofluoroalkane; TID, three times daily; ICS, inhaled corticosteroids. 
$180 \mu \mathrm{g}$, groups, respectively), upper respiratory tract infection ( 3 and 5 subjects in the placebo and albuterol HFA $180 \mu \mathrm{g}$ groups, respectively) and sinus tachycardia $(2,2$, and 5 subjects in the placebo, albuterol HFA $90 \mu \mathrm{g}$ and $180 \mu \mathrm{g}$ groups, respectively) and teething $(3,4$, and 1 subjects in the placebo and albuterol HFA $90 \mu \mathrm{g}$ and $180 \mu \mathrm{g}$ groups, respectively). One subject in the placebo group experienced a serious adverse event (asthma exacerbation) which occurred 28 days after study medication administration and was considered by the investigator to be moderate and most likely secondary to subject's viral upper respiratory tract infection with no causal association with study drug. This patient withdrew from the study due to lack of efficacy.

No clinically significant differences were observed between treatment groups in laboratory evaluations, vital signs or physical examination. Although there were a few shifts to high or low in blood glucose or serum potassium levels, no cases of drug-related abnormalities were reported in the albuterol HFA treated groups. Adrenergic stimulation was assessed using 7 individual items (sleep well, content/ cheerful, act moody, unusually irritable, sleep through the night, unusually difficult and react by crying) from the FSII(R) questionnaire. Higher scores reflected a better functional status. Mean scores increased for all items in each treatment group with the exception of the "content/cheerful" score, which remained high and unchanged, and the score for "unusually irritable and difficult" that changed slightly in all treatment groups. No evidence of adrenergic stimulation was derived from these scores.

\section{Cardiovascular Safety}

Across all groups, $82-86 \%$ subjects had normal ECGs at screening. Electrocardiograms were evaluated by a central cardiologist as normal, abnormal not clinically significant or abnormal clinically significant. Additionally ECGs at randomization visit 2 post-dose, and week 4 pre and postdose were compared for clinically significant changes with respect to screening or randomization pre-dose or week 4 pre-dose. Of the 8 subjects that prematurely discontinued the study, 6 had ECGs performed, none of which were abnormal when compared with baseline measurements.

At screening (Visit 1), 1 subject in the placebo group, (sinus bradycardia), 4 subjects in the albuterol HFA $90 \mu \mathrm{g}$ group (1 with sinus rhythm and biventricular hypertrophy, 1 with right ventricular hypertrophy and 2 with sinus tachycardia) and 2 subjects (sinus tachycardia) in the $180 \mu \mathrm{g}$ group had abnormal clinically significant ECGs.

At randomization visit 2 post-dose six subjects each in the placebo (4 with sinus tachycardia, 1 with sinus rhythm non-specific T-wave abnormality and 1 with low right atrial rhythm abnormality and sinus tachycardia) and albuterol $180 \mu \mathrm{g}$ group (5 with sinus tachycardia of which 1 also had left axis deviation and 1 subject had sinus bradycardia) and 3 subjects in the albuterol $90 \mu \mathrm{g}$ ( 1 each with sinus tachycardia or bradycardia and 1 with sinus tachycardia and $1^{\text {st }}$ degree AV block) showed a clinically significant change as compared to visit 2 pre-dose

At Week 4 visit pre-dose, five subjects in the albuterol HFA $180 \mu \mathrm{g}$ group had a clinically significant change in ECG when compared to visit 2 pre-dose (4 subjects had sinus tachycardia 1 subject had bradycardia) One subject in the placebo group had a clinically significant prolonged QT which resolved at Week 4 post dose.

Pre and post dose ECG comparisons at week 4 showed ten subjects, five in each of the albuterol HFA $90 \mu \mathrm{g}$ and $180 \mu \mathrm{g}$ groups and none in the placebo group with a clinically significant change in ECG. In the albuterol $90 \mu \mathrm{g}$ three subjects had ECG voltage measurements consistent with ventricular hypertrophy as determined by the independent cardiologist [either right, left (with sinus arrhythmia) or biventricular hypertrophy with right axis deviation]. Two of these subjects had normal findings with a repeat ECG and ECG cardiology consult. The third subject was contacted but did not return for follow-up. One subject had sinus arrhythmia (potentially drug-related) that resolved in a week and 1 subject had increase PR interval. In the albuterol $180 \mu \mathrm{g}$ group 4 subjects had tachycardia and 1 subject had sinus bradicardia. None of the reported eposides of tachycardia was felt by the investigators to be related to albuterol HFA treatment.

No subjects had a QT or QTc interval $>460$ msec. Mean heart rate, QT and PR intervals and QRS durations summarized in Table 2, were similar to baseline values at week 4. No significant differences were observed in drug related adverse events that occurred during the study.

\section{Efficacy}

Although this study was not powered for efficacy, changes from baseline to endpoint in efficacy parameters were evaluated. At screening, mean 24-hour asthma symptom scores were low (noticeable symptoms, but no interference with daily activities or sleep) and similar across treatment groups. Asthma scores declined slightly in each group over 4 weeks of treatment. No difference between the two treatment groups was observed in daytime and nighttime symptom scores. All groups showed a favorable change in the percentage of symptom free 24-hour days, rescue albuterol use and nighttime awakenings compared to baseline; however the differences were not significant between treatment groups.

\section{DISCUSSION}

Albuterol sulfate inhalation aerosol is well established in the treatment and prevention of reversible obstructive airways disease and is currently approved in children over 4 years of age. However, there is limited information available for albuterol HFA in younger children. This study extends the safety profile of albuterol HFA for the treatment of respiratory symptoms in children birth to $<24$ months of age.

Asthma treatment guidelines state that metered-dose inhaler with auxiliary devices and face masks can be used in children less than 4 years of age and recommend nebulizers for children under 24 months of age and children of all ages who cannot use MDIs with auxiliary devices [5, 6]. Recent studies have shown the metered-dose inhaler with spacer to be an effective alternative to nebulizers in the administration of beta $_{2}$-agonists for the treatment of children with severe or potentially severe acute asthma in the emergency department $[14,15]$. MDI with valved holding chamber offers practical advantages including less set-up time, portability, the capacity for home use, and no need for daily disinfection [16-19]. Furthermore medication delivery with a valved 
Table 2. Summary of ECG Measures, Mean (Change from Baseline)

\begin{tabular}{|c|c|c|c|}
\hline ECG Measure & $\begin{array}{c}\text { Placebo } \\
(N=28)\end{array}$ & $\begin{array}{c}\text { Albuterol HFA } \\
90 \mu \mathrm{g} \text { TID }(N=29)\end{array}$ & $\begin{array}{c}\text { Albuterol HFA } \\
180 \mu \mathrm{g} \text { TID }(N=29)\end{array}$ \\
\hline \multicolumn{4}{|l|}{ Heart Rate (bpm) } \\
\hline Baseline $^{a}$ & 127.2 & 132.4 & 128.6 \\
\hline Week 4 Pre-dose ${ }^{c}$ & $126.4(-2.0)$ & $126.3(-5.4)$ & $128.1(-1.6)$ \\
\hline \multicolumn{4}{|l|}{ QT Interval (msec) } \\
\hline Baseline $^{a}$ & 279.1 & 275.4 & 273.4 \\
\hline Week 4 Pre-Dose ${ }^{c}$ & $279.8(2.3)$ & $279.1(0.7)$ & $274.9(1.0)$ \\
\hline Week 4 Post-dose ${ }^{c}$ & $279.0(1.5)$ & $279.0(2.3)$ & $276.1(2.3)$ \\
\hline \multicolumn{4}{|l|}{ QTc Interval ${ }^{\mathrm{b}}$ (msec) } \\
\hline Week 4 Post-dose ${ }^{c}$ & $354.0(-2.0)$ & $358.2(-0.7)$ & $358.5(6.3)$ \\
\hline \multicolumn{4}{|l|}{ PR Interval (msec) } \\
\hline Baseline $^{a}$ & 111.6 & 116.5 & 111.3 \\
\hline Week 4 Pre-Dose & 115.4 & 118.5 & 110.7 \\
\hline Week 4 Post-dose & 114.4 & 118.2 & 114.1 \\
\hline \multicolumn{4}{|l|}{ QRS Duration (msec) } \\
\hline Baseline $^{a}$ & 67.5 & 66.2 & 66.2 \\
\hline Week 4 Pre-Dose & 68.3 & 67.4 & 66.3 \\
\hline
\end{tabular}

HFA, hydrofluoroalkane; TID, three times daily.

${ }^{a}$ Baseline, value obtained at Screening Visit.

${ }^{\mathrm{b}}$ Corrected for heart rate using Fredericia's formula.

${ }^{\mathrm{c}}$ Value is Raw Value and (Change from Baseline).

holding chamber and spacer provides a greater distribution throughout the lungs due to smaller particle production, and significantly reduced amounts of large particle deposition in the oropharynx $[20,21]$.

The doses selected in this study were similar to recommended doses per the Ventolin ${ }^{\circledR}$ HFA package label and are consistent with asthma treatment guidelines, the doses used for nebulization and normal clinical practice [5, $18,22,23]$. The medications were given three times a day for 29 days as part of the study design in order to make dosing easier on the care giver and to better assess safety.

Studies of inhaled bronchodilator safety in children younger than 24 months old are limited. In a comparative study, children 2 years and older with asthma exacerbations treated with beta-agonists via a MDI and spacer required shorter treatment times in the emergency department, and smaller increases in heart rate than those treated with a nebulizer [24]. Another recent study in children less than 5 years of age demonstrated a significant heart rate increase in the salbutamol aerosol therapy via a jet nebulizer when compared to the MDI with spacer [25].

Electrocardiographic adverse effects have been reported with the use of beta-agonists in adults and children. The stimulation of the cardiac $\beta$-adrenergic receptors could cause dose related pharmacologically predictable adverse effects [26] such as tachycardia, and arrhythmias [27, 28]. $\beta_{2}$ mediated electrolyte disturbances include hypokalemia from increased cellular influx of potassium into cells and hyperglycemia due to increased glycogenolysis [27-29]. A 14 day study with high dose (1000 $\mu \mathrm{g} 4$ times daily) inhaled albuterol treatment demonstrated that improvement in bronchodilatation $\left(\mathrm{FEV}_{1}\right.$, maximal $\left.\Delta \mathrm{FEV}_{1}\right)$ is maintained and that there was attenuation in systemic adverse effects (glucose or potassium abnormalities, tremor and palpitations) [30]. A recent study of children 5 to 17 years old attributes the higher and significant change in heart rate after nebulizer aerosol treatment to greater deposition, pooling and delayed absorption of albuterol in the upper airway [31]. Another study showed no significant increase in QTc prolongation in adult subjects with acute asthma exacerbations treated with doses of 400 or $600 \mu \mathrm{g}$ salbutamol via MDI with spacer at 10 -minute intervals over 3 hours [32]. In a recent study in 2-4 year old children treated with either albuterol HFA $90 \mu \mathrm{g}$ or $180 \mu \mathrm{g}$ or placebo 3 times a day for 4 weeks, there were no clinically relevant differences in the mean change from baseline in the QT interval between treatment groups [12].

In our study, children aged birth to $<24$ months with a history of bronchospasm treated with albuterol HFA MDI 
$90 \mu \mathrm{g}$ or $180 \mu \mathrm{g}$ with a valved holding chamber three times daily for 4 weeks, demonstrated no clinically relevant safety issues. The overall incidence of adverse events possibly related to adrenergic stimulation in the current study was low across the treatment groups and did not cause subject withdrawal from study. Episodes of sinus tachycardia were slightly higher in the albuterol $180 \mu \mathrm{g}$ group, almost half of which were not considered clinically significant by the independent cardiologist and those that were clinically significant were subjects in distress who were crying during the ECG procedure. Three subjects exhibited ECG voltage measurements suggestive of ventricular hypertrophy. These were not confirmed upon repeat ECG measurements or consultation. Electrolyte imbalances such as hypokalemia and hyperglycemia were not experienced with albuterol HFA treatment. There were no clinically relevant differences between treatment groups in cardiovascular parameters such as, blood pressure, or other ECG findings over the treatment period. Albuterol HFA treatment demonstrated a slight improvement in most efficacy parameters, like the mean 24hour asthma symptom score, percentage of symptom free 24hour days, rescue albuterol use, and nighttime awakenings though none were significantly different from placebo. Statistical differences were not demonstrated for the efficacy outcomes primarily due to the study not being powered to show differences and mildness of the subject symptoms at screening.

Although abuterol HFA is currently not indicated for use in children less than 4 years of age, it is recognized that physicians sometimes prescribe this medication in this younger age population. As there are very few studies in children less than 2 years of age, the aim of this study was to examine the safety of albuterol HFA MDI in this age group. Due to the uniqueness of this population and the challenges of safety parameter collection, a patient may or may not experience the events observed in this study. Caution needs to be exercised in prescribing the appropriate therapy because of potential complications during an episode of bronchospasm in this age group. Moreover, monitoring the patient closely during treatment and providing education for the proper use of MDI-spacer-facemask combination is also of utmost importance. In conclusion, this study in children less than 24 months old with a history of symptoms compatible with bronchospasm demonstrated no clinically relevant safety signals during prolonged patient treatment in either treatment group.

\section{TRIAL REGISTRATION}

www.gsk-clinicalstudyregister.com; SBO30001

\section{ACKNOWLEDGEMENTS}

Declaration of Interest: This study is sponsored by GlaxoSmithKline.

We also thank the SBO30001 investigators in the United States who provided valuable data by study participation.

Drs. Hedrick, Baker, Atlas and Naz are investigators for GlaxoSmithKline. Mr. Lincourt, Ms. Trivedi, Mrs. Ellsworth and Dr. Davis are full-time employees of GlaxoSmithKline.

\section{REFERENCES}

[1] American Lung Association. Epidemiology \& Statistics Unit, Research and Program Services. Trends in Asthma Morbidity and Mortality. [Accessed on: January 2009] Available at: www.lungusa.org.

[2] Bloom B, Dey AN. Summary health statistics for U.S. children, national health interview survey, 2004. Vital Health Stat 2006; 10(227): 1-94.

[3] Pediatric Asthma Committee. Pediatric Asthma: Promoting Best Practice Guide for Management of Asthma in Children. Milwaukee, WI: American Academy of Allergy, Asthma and Immunology; copyright 1999-2004.

[4] National Institutes of Health. National Asthma Education and Prevention Program (NAEPP). The NAEPP Expert Panel Report 3 (EPR-3) Summary Report 2007: Guidelines for the Diagnosis and Management of Asthma. US Department of Health and Human Services; National Institutes of Health. October 2007; NIH Publication No. 08-5846.

[5] Global Initiative for Asthma (GINA). Global Strategy for Asthma Management and Prevention. [Updated 2007]. Available at: www.ginasthma.org

[6] Delgado A, Chou KJ, Silver EJ, Crain EF. Nebulizers vs metereddose inhalers with spacers for bronchodilator therapy to treat wheezing in children aged 2 to 24 months in a pediatric emergency department. Arch Pediatr Adolesc Med 2003; 157: 76-80.

[7] Castro-Rodriguez JA, Rodrigo GJ. $\beta$-agonists through metereddose inhaler with valved holding chamber versus nebulizer for acute exacerbation of wheezing or asthma in children under 5 years of age: A systematic review with meta-analysis. J Pediatr 2004; 145: 172-7.

[8] Leversha AM, Campanella SG, Aickin RP, Asher MI. Costs and effectiveness of spacer versus nebulizer in young children with moderate and severe acute asthma. J Pediatr 2000; 136: 497-502.

[9] Fink JB, Rubin BK. Problems with inhaler use: a call for improved clinician and patient education. Respir Care 2005; 50(10): 1360-74.

[10] Dolovich MB, Ahrens RC, Hess DR, et al. American college of chest physicians; American college of asthma, allergy, and immunology device selection and outcomes of aerosol therapy: evidence-based guidelines: American college of chest physicians/American college of asthma, allergy, and immunology. Chest 2005; 127(1): 35-71.

[11] Tal A, Golan H, Grauer N, Aviram M, Albin D, Quastel MR. Deposition pattern of radiolabeled salbutamol inhaled from a metered dose inhaler by means of a spacer with mask in young children with airway obstruction. J Pediatr 1996; 128: 479-84.

[12] Kerwin EM, Tarpay MM, Kim K, et al. Safety and efficacy of a new albuterol hydrofluoroalkane formulation for treating asthma symptoms in children two to less than four years of age. Pediatr Asthma Allergy Immunol 2006; 19(2): 81-9.

[13] Stein RE, Jessop DJ. Functional status II(R): a measure of child health status. Med Care 1990; 28:1041-55.

[14] Hendeles L, Hatton RC, Coons TJ, Carlson L. Automatic replacement of albuterol nebulizer therapy by metered-dose inhaler and valved holding chamber. Am J Health Syst Pharm 2005; 62:1053-61.

[15] Sannier S, Timsit B, Cojocaru A, et al. Metered-dose inhaler with spacer vs nebulization for severe and potentially severe acute asthma treatment in the pediatric emergency department. Arch Pédiatr 2006; 13(3): 238-44.

[16] Barnes KL, Clifford R, Holgate ST, Murphy D, Comber P, Bell E. Bacterial contamination of home nebulizers. Br Med J 1987; 95: 812 .

[17] Bowton DL, Goldsmith WM, Haponik EF. Substitution of metered dose inhalers for hand held nebulizers. Success and cost savings in a large, acute care hospital. Chest 1992; 101: 305-8.

[18] Blake KV, Hoppe M, Harman E, Hendeles L. Relative amount of albuterol delivered to lung receptors from a metered dose inhaler and nebulizer solution. Bioassay by histamine bronchoprovocation. Chest 1992; 101: 309-15

[19] Summer W, Elston R, Tharpe L, Nelson S, Haponik EF. Aerosol bronchodilator delivery methods. Relative impact on pulmonary function and cost of respiratory care. Arch Intern Med 1989; 149: 618-23. 
[20] Newman SP, Millar AB, Lennard-Jones TR, Moren F, Clarke SW. Improvement of pressurized aerosol deposition with nebuhaler spacer device. Thorax 1984; 39: 933-4.

[21] Newhouse MT, Dolovich MB. Control of asthma by aerosols. N Engl J Med 1986; 315: 870-4.

[22] Chou KJ, Cunningham SJ, Crain EF. Metered-dose inhalers with spacers $v s$ nebulizers for pediatric asthma. Arch Pediatr Adolesc Med 1995; 149: 201-5.

[23] Chandra A, Shim C, Hillel W, et al. Regular vs ad-lib albuterol for patients hospitalized with acute asthma. Chest 2005; 128: 1115-20.

[24] Deerojanawong J, Manuyakorn W, Prapphal N, Harnvuthakorn C, Sritippayawan S, Samransamruajkit R. Randomized controlled trial of salbutamol aerosol therapy via metered dose inhaler-spacer $v s$ jet nebulizer in young children with wheezing. Pediatr Pulmonol 2005; 39: 466-72.

[25] Clark DJ, Lipworth BJ. Dose-response of inhaled drugs in asthma. Clin Pharmacokinet 1997; 32: 58-74.

[26] Tandon MK. Cardiopulmonary effects of fenoterol and salbutamol aerosols. Chest 1980; 77: 429-431.

[27] Wong CS, Pavord ID, Williams J, Britton JR, Tattersfield AE. Bronchodilator, cardiovascular and hypokalemic effects of fenoterol, salbutamol and terbutaline in asthma. Lancet 1990; 336:1396-9.

[28] Bremner P, Woodman K, Burgess C, et al. A comparison of the cardiovascular and metabolic effects of formoterol, salbutamol and fenoterol. Eur Respir J 1993; 6: 204-10.

[29] Lipworth BJ, McDevitt DG. Beta-adrenoceptor responses to inhaled salbutamol in normal subjects. Eur J Clin Pharmacol 1989; 36: 239-45.

[30] Lipworth BJ, Struthers AD, McDevitt DG. Tachyphylaxis to systemic but not to airway responses during prolonged therapy with the high dose inhaled salbutamol in asthmatics. Am Rev Respir Dis 1989; 140: 586-92.

[31] Schuh S, Johnson DW, Stephens D, Callahan S, Winders P, Canny GJ. Comparison of albuterol delivered by a metered dose inhaler with spacer versus a nebulizer in children with mild acute asthma. J Pediatr 1999; 135: 22-7.

[32] Rodrigo G, Rodrigo C. Metered dose inhaler salbutamol treatment of asthma in the ED: comparison of two doses with plasma levels. Am J Emerg Med 1996; 14: 144-50.

(C) Hedrick et al.; Licensee Bentham Open.

This is an open access article licensed under the terms of the Creative Commons Attribution Non-Commercial License (http://creativecommons.org/licenses/by$\mathrm{nc} / 3.0 /$ ) which permits unrestricted, non-commercial use, distribution and reproduction in any medium, provided the work is properly cited. 